Vol. 36, Issue 3, June 2009

Editorial

154 Molecular Blood Group Diagnostics

Gassner, C. (Innsbruck)

Review Articles

157 Microarray Beads for Identifying Blood Group Single Nucleotide Polymorphisms Drago, F.; Karpasitou, K.; Poli, F. (Milan)

162 The Bloodgen Project of the European Union, 2003-2009

Avent, N.D. (Bristol); Martinez, A. (Derio); Flegel, W.A. (Ulm); Olsson, M.L. (Lund); Scott, M.L. (Bristol); Nogués, N. (Barcelona); Písačka, M. (Prague); Daniels, G.F. (Bristol); Muñiz-Diaz, E. (Barcelona); Madgett, T.E. (Bristol); Storry, J.R. (Lund); Beiboer, S.; Maaskant-van Wijk, P.M. (Amsterdam); von Zabern, I. (Ulm); Jiménez, E.; Tejedor, D.; López, M. (Derio); Camacho, E. (Barcelona); Cheroutre, G. (Amsterdam); Hacker, A. (Ulm); Jinoch, P.; Svobodova, I. (Prague); van der Schoot, E.; de Haas, M. (Amsterdam)

168 Applications and Experience with PCR-Based Assays to Predict Blood Group Antigens Reid, M.E. (New York, NY)

181 Molecular Diagnostics in Transfusion Medicine: In Capillary, on a Chip, in Silico, or in Flight? Garritsen, H.S.P. (Braunschweig); Fan, A. (Basel); Lenz, D.; Hannig, H. (Braunschweig); Zhong, X.Y. (Basel); Geffers, R.; Lindenmaier, W.; Dittmar, K.E.J.; Wörmann, B. (Braunschweig)

189 Prenatal RhD Testing:

A Review of Studies Published from 2006 to 2008 Legler, T.J.; Müller, S.P.; Haverkamp, A. (Göttingen); Grill, S.; Hahn, S. (Basel)

199 Screening Donors for Rare Antigen Constellations Wagner, F.F. (Springe)

204 Sequence-Based Typing of Human Blood Groups Seltsam, A. (Springe); Doescher, A. (Oldenburg)

\section{Band 36, Heft 3, Juni 2009}

Editorial

154 Molekulare Blutgruppendiagnostik Gassner, C. (Innsbruck)

Übersichtsarbeiten

157 Microarray-Beads zur Identifizierung von Blutgruppen-Single-Nucleotide-Polymorphisms Drago, F.; Karpasitou, K.; Poli, F. (Milan)

162 Das Bloodgen-Projekt der Europäischen Union 2003-2009

Avent, N.D. (Bristol); Martinez, A. (Derio); Flegel, W.A. (Ulm); Olsson, M.L. (Lund); Scott, M.L. (Bristol); Nogués, N. (Barcelona); Písačka, M. (Prague); Daniels, G.F. (Bristol); Muñiz-Diaz, E. (Barcelona); Madgett, T.E. (Bristol); Storry, J.R. (Lund); Beiboer, S.; Maaskant-van Wijk, P.M. (Amsterdam); von Zabern, I. (Ulm); Jiménez, E.; Tejedor, D.; López, M. (Derio); Camacho, E. (Barcelona); Cheroutre, G. (Amsterdam); Hacker, A. (Ulm); Jinoch, P.; Svobodova, I. (Prague); van der Schoot, E.; de Haas, M. (Amsterdam)

168 Anwendungen und Erfahrungen mit PCR-basierten Assays zur Bestimmung von Blutgruppenantigenen Reid, M.E. (New York, NY)

181 Molekulare Diagnostik in der Transfusionsmedizin: in der Kapillare, auf dem Chip, in silico oder im Vorbeigehen?

Garritsen, H.S.P. (Braunschweig); Fan, A. (Basel); Lenz, D.; Hannig, H. (Braunschweig); Zhong, X.Y. (Basel); Geffers, R.; Lindenmaier, W.; Dittmar, K.E.J.; Wörmann, B. (Braunschweig)

189 Pränatale Bestimmung des RhD-Status:

Ein Review von Studien, die von 2006 bis 2008 publiziert wurden

Legler, T.J.; Müller, S.P.; Haverkamp, A. (Göttingen); Grill, S.; Hahn, S. (Basel)

199 Suche nach Spendern mit seltenen Antigenkonstellationen Wagner, F.F. (Springe)

204 Sequenzierung menschlicher Blutgruppen Seltsam, A. (Springe); Doescher, A. (Oldenburg)

\section{KARGER}

Fax +497614520714

Information@Karger.de

www.karger.com 
Vol. 36, Issue 3, June 2009

Original Articles

213 Molecular Bases and Genotyping for Rare Blood Types

Jungbauer, C. (Wien)

219 Application of a Multivariant, Caucasian-Specific, Genotyped Donor Panel for Performance Validation of MDmulticard $^{\circledR}$, ID-System ${ }^{\circledR}$, and Scangel ${ }^{\circledR}$ RhD/ABO Serotyping

Gassner, C.; Rainer, E.; Pircher, E.; Markut, L. (Innsbruck); Körmöczi, G.F.; Jungbauer, C. (Wien); Wessin, D. (Feldkirch); Klinghofer, R. (Mistelbach); Schennach, H. (Innsbruck); Schwind, P. (Düdingen); Schönitzer, D. (Innsbruck)

\section{Commentaries}

226 Will Genotyping Replace Serology in Future Routine Blood Gouping?

Opinion 1

Wagner, F.F. (Springe)

228 Will Genotyping Replace Serology in Future Routine Blood Grouping?

Opinion 2

Hustinx, H.; Fontana, S.; Gowland, P.; Niederhauser, C. (Bern)

230 Will Genotyping Replace Serology in Future Routine Blood Grouping?

Opinion 3

Schönitzer, D. (Innsbruck)

232 Will Genotyping Replace Serology in Future Routine Blood Grouping? Opinion 4. Personalized versus Universal Blood Transfusions - Combining the Efforts Storry, J.R.; Olsson, M.L. (Lund)

234 Will Genotyping Replace Serology in Future Routine Blood Grouping? - Opinion 5 van der Schoot, C.E.; Veldhuisen, B.; de Haas, M. (Amsterdam)

237 News / Ticker

238 Meetings and Conferences

240 Guidelines for Authors

161 Imprint

\section{Band 36, Heft 3, Juni 2009}

Originalarbeiten

213 Molekulare Grundlagen und Genotypisierung für seltene Blutgruppen

Jungbauer, C. (Wien)

219 Verwendung eines multivarianten, indoeuropäischspezifischen und genotypisierten Spender-Panels für den Vergleich der MDmulticard ${ }^{\circledR}$-, ID-System ${ }^{\circledR}$ - und Scangel ${ }^{\circledR}$-RhD/ABO-Serotypisierung

Gassner, C.; Rainer, E.; Pircher, E.; Markut, L. (Innsbruck); Körmöczi, G.F.; Jungbauer, C. (Wien); Wessin, D. (Feldkirch); Klinghofer, R. (Mistelbach); Schennach, H. (Innsbruck); Schwind, P. (Düdingen); Schönitzer, D. (Innsbruck)

\section{Kommentare}

226 Wird die Genotypisierung die Serologie in der zukünftigen Routine-Blutgruppenbestimmung ersetzen? Meinung 1 Wagner, F.F. (Springe)

228 Wird die Genotypisierung die Serologie in der zukünftigen Routine-Blutgruppenuntersuchung ersetzen? Meinung 2 Hustinx, H.; Fontana, S.; Gowland, P.; Niederhauser, C. (Bern)

230 Wird die Genotypisierung die Serologie in der zukünftigen Routine-Blutgruppenuntersuchung ersetzen? Meinung 3

Schönitzer, D. (Innsbruck)

232 Wird die Genotypisierung die Serologie in der künftigen Routine-Blutgruppenbestimmung ersetzen? Meinung 4. Personalisierte versus universelle Bluttransfusionen - Verbindung der Anstrengungen Storry, J.R.; Olsson, M.L. (Lund)

234 Wird die Genotypisierung die Serologie in der künftigen Routine-Blutgruppenbestimmung ersetzen? Meinung 5 van der Schoot, C.E.; Veldhuisen, B.; de Haas, M. (Amsterdam)

237 News / Ticker

238 Tagungen und Kongresse

240 Hinweise für Autoren

161 Impressum

\section{KARGER}

Fax +49761 4520714

Information@Karger.de

www.karger.com
(C) 2009 S. Karger GmbH, Freiburg 